\title{
LA EVOLUCIÓN DEL LÉXICO DISPONIBLE EN ESPAÑOL COMO LENGUA EXTRANJERA EN APRENDICES MARROQUÍES DE ENSEÑANZA SECUNDARIA Y UNIVERSITARIA
}

\author{
Mohamed Serfati \\ Universidad de Las Palmas de Gran Canaria (España) \\ serfati18@gmail.com
}

Recibido: 06/08/2020 - Aprobado: 30/09/2020 - Publicado: 15/04/2021

DOI: doi.org/10.17533/udea.lyl.n79a09

Resumen: Este estudio presenta el análisis de la disponibilidad léxica de los aprendices marroquíes de ELE de nivel secundario y universitario de la región Sus-Masa —Agadir, Marruecos— basándose en las pautas metodológicas propuestas por el Proyecto Panhispánico de Disponibilidad Léxica (PPHDL). El objetivo es cotejar los resultados y presentar algunas consideraciones cuantitativas sobre los mismos, a saber, la producción de vocablos, el promedio de respuestas por informante y el grado de densidad. Los resultados globales revelaron que existe una diferencia estadísticamente significativa en el caudal léxico de los alumnos de secundaria y el de los estudiantes universitarios.

Palabras clave: ELE, léxico disponible; caudal léxico; competencia léxica; aprendices marroquíes; estudiantes de secundaria y universidad.

\section{EVOLUTION OF THE AVAILABLE LEXICON IN SPANISH AS A FOREIGN LANGUAGE OF MOROCCAN LEARNERS OF SECONDARY AND UNIVERSITY EDUCATION}

\begin{abstract}
This study presents the analysis of the lexical availability of Moroccan secondary and university level learners of Spanish as a Foreign Language from the Souss-Massa region -Agadir, Morocco-, based on the methodological guidelines proposed by the Pan-Hispanic Project of Lexical Availability (PPHDL). The objective is to compare the results from both levels and to present some quantitative considerations about them, namely, the production of words, the average of responses per informant and the degree of density. The results revealed that there is a statistically significant difference in the lexical flow of high school students and that of university students.
\end{abstract}

Key words: Spanish as a foreign language; available lexicon; lexical flow; lexical competence; Moroccan apprentices; high school and college students. 


\section{Introducción}

$\mathrm{L}$

os estudios de disponibilidad léxica fueron iniciados en Francia a mediados del siglo Xx por un grupo de lingüistas galos - Gougenheim, Michéa, Rivenc \& Sauvageot, 1956- que buscaban facilitar la enseñanza del francés a extranjeros en las excolonias de la llamada Unión Francesa. Fue Michéa (1953) quien dio a conocer por vez primera este nuevo concepto y su definición:

Un mot disponible est un mot qui, sans être particulièrement fréquent, est cependant toujours prêt à être employé et se présente immédiatement et naturellement à l'esprit au moment où l'on en a besoin. C'est un mot qui, faisant partie d'associations d'idées usuelles, existe en puissance chez le sujet parlant, dès que ces associations entrent en jeu. (p. 340).

[Una palabra disponible es aquella que, sin ser particularmente frecuente, está siempre lista para ser empleada y viene inmediata y naturalmente a la mente en el momento en que se tiene necesidad de ella. Es una palabra que, formando parte de las asociaciones de ideas usuales, existe en potencia en el sujeto hablante en cuanto estas asociaciones entran en juego.] (p. 340).

En el mundo hispánico, el inicio de estos estudios vino de la mano de López Morales en sus primeros hallazgos de 1973, 1978 y 1979 al analizar el léxico disponible en español de los escolares de San Juan en Puerto Rico. Posteriormente, muchos estudiosos seguidores de tales avances sintieron interés por este ámbito de investigación y trabajaron el léxico disponible en el español de muchas comunidades hispanohablantes (Samper Padilla, Bellón Fernández \& Samper Hernández, 2003).

En este sentido, este trabajo gira en torno a la disponibilidad léxica de los aprendices marroquíes en español como lengua extranjera. Es un estudio que se inscribe en el marco de los trabajos de léxico disponible en ELE que se realizan en diferentes países del mundo hispánico. Estos estudios constituyen una vertiente investigativa que se desarrolla paralelamente a las directrices del Proyecto Panhispánico de Disponibilidad Léxica (PPHDL), que coordina el profesor López Morales. Se trata de un macroproyecto que tiene por objeto recopilar el caudal léxico tanto de hablantes nativos como de aprendices de ELE y realizar posteriormente no solo un análisis de índole cuantitativa, sino también estudios cualitativos sobre cuáles aspectos léxicos son mencionados por los informantes cuando estos piensan en determinados temas, y hasta qué punto ese lexicón mental es un verdadero reflejo de la realidad social de los hablantes.

De esta forma, este estudio tiene como objetivo recopilar y analizar cuantitativamente el caudal léxico que tienen a su disposición los aprendices marroquíes que aprenden español en la enseñanza secundaria y universitaria. A la hora de valorar los resultados globales en lo correspondiente a la producción léxica en los dos niveles investigados, se parte de la hipótesis de que cuanto más alto sea el nivel educativo del discente, mayor será su competencia léxica. ${ }^{1}$ Por lo tanto, es de suponer que los aprendices marroquíes de nivel superior incorporarán más

1. Según Marconi (2000), la competencia léxica es una familia de destrezas complejas que no se limita a conocer el significado de una palabra. De esta forma, se pueden diferenciar dos elementos importantes. Por una parte, la capacidad del individuo para almacenar palabras y por otra, las habilidades para producirlas. 
La evolución del léxico disponible en español como lengua extranjera en aprendices marroquíes vocabulario que los alumnos de secundaria.

\section{Estado del arte}

Actualmente, los estudios sobre el léxico disponible en español como lengua extranjera han sido abundantes. Prueba de ello son los trabajos pioneros de Carcedo González (2000) en Finlandia y Samper Hernández (2002) en Salamanca, España. Desde estos primeros estudios, esta nueva corriente investigativa no para de crecer y el ritmo de trabajo y de publicaciones sigue incrementando vertiginosamente (López González, 2013, 2014). Se engrosaron, pues, los estudios dedicados al análisis del léxico disponible en español que aprenden los extranjeros en diferentes países del mundo: Jing (2006) e Hidalgo Gallardo (2018) en China; Medina Arejita (2009) en Alemania; Sifrar Kalan (2009) en Eslovenia; López González (2010) en Polonia; Camarena Ortiz (2010) en México; Fernández Leyva (2013) en Cuba; González Fernández (2013) en Turquía; Serfati y Aabidi (2013) en Marruecos; Sandu (2014) en Rumanía; Pedroni Torres (2015) en Brasil; Blanco-San Martín y Ferreira Cabrera (2018) en Chile; Mendoza Puertas (2018) en Corea del Sur; Rubio Lastra (2020) en Taiwán; entre otros, que se fijan los mismos objetivos basándose, claro está, en los presupuestos metodológicos del Proyecto Panhispánico de Disponibilidad Léxica (PPHDL). Por otra parte, otros estudios similares fueron llevados a cabo en plena inmersión lingüística — en España, con alumnos de distintas procedencias — por estudiosos tales como López Rivero (2008), Pérez Serrano (2009) y Gallego Gallego (2014) en Madrid; Sánchez-Saus Laserna (2016) en Andalucía, entre otros. Dentro de este grupo se incluyen también los estudios con inmigrantes no hispanohablantes, tanto adultos (Fernández-Merino, 2013) como escolares (Jiménez Berrio, 2013), y demás.

\section{Metodología}

\subsection{Participantes}

La muestra de este estudio descriptivo está constituida por un total de 350 aprendices marroquíes de secundaria (Aabidi, 2020) y 350 universitarios del Departamento de Filología Hispánica inscritos en la Facultad de Letras y Ciencias Humanas de la Universidad Ibn Zohr en Agadir (Serfati, 2016). Los aprendices de secundaria realizan sus estudios en tres niveles escolares: inicial — 90 alumnos — , intermedio — 120 encuestados — y avanzado — 140 informantes — 22 en tres Academias Regionales de Educación y Formación (AREF); ${ }^{3}$ mientras que los universitarios están distribuidos como sigue: curso 1 -142 encuestados —, curso 2 - 96- y curso 3 - 112 informantes-. Además, en el caso de secundaria, los alumnos pertenecen a dos zonas geográficas distintas: alumnos de excolonia

2. Los niveles educativos contemplados son Tronco Común (TC), Primero de Bachillerato - $1 .^{\circ}$ Bach.- - y Segundo de Bachillerato -2..$^{\circ}$ Bach._- que corresponden más o menos a los niveles de referencia A1, A2 y B1 del Marco Común Europeo de Referencia (MCER).

3. Las Academias Regionales de Educación y Formación (AREF) donde se han recopilado los datos son Souss-Massa, El AaiúnSaguia El Hamra y Dakhla-Oued Ed Dahab. 
La evolución del léxico disponible en español como lengua extranjera en aprendices marroquíes

francesa - 125, el 35,71\% de los encuestados - y discentes de excolonia española — 225, el 64,28 \% de los informantes - Mientras que, en la universidad, todos los estudiantes pertenecen — en el momento de la recogida de los datos - al Departamento de Lengua y Literatura Hispánicas en la Facultad de Letras y Ciencias Humanas de la Universidad Ibn Zohr en Agadir. Así pues, para el análisis de los datos es necesario comenzar a destacar las características más importantes que hacen diferentes los dos trabajos.

\begin{tabular}{|c|c|c|}
\hline Características & Secundaria & Universidad \\
\hline Encuestas & 350 & 350 \\
\hline Centros de interés & 17 & 17 \\
\hline Variables analizadas & $\begin{array}{c}\text { Sexo } \\
\text { Lengua materna } \\
\text { Zona geográfica } \\
\text { Nivel educativo } \\
\text { Nivel sociocultural }\end{array}$ & $\begin{array}{c}\text { Sexo } \\
\text { Lengua materna } \\
\text { Curso académico } \\
\text { Nivel sociocultural } \\
\text { Conocimiento de otra(s) } \\
\text { lengua(s) }\end{array}$ \\
\hline $\begin{array}{ll}\text { Tiempo para rellenar la } \\
\text { encuesta }\end{array}$ & 2 minutos & 2 minutos \\
\hline Edad de los informantes & De 15 a 18 años & De 18 a 24 años \\
\hline
\end{tabular}

Tabla 1. Antecedentes de las dos muestras. Fuente: elaboración propia.

\subsection{Instrumento}

El trabajo objeto de estudio se ha basado en un test asociativo que está constituido por 17 centros de interés ${ }^{4}$, lo que ha permitido acceder directamente a los informantes y recoger datos de interés que se presentan a lo largo del análisis. Los aprendices escribieron a continuación de las líneas trazadas en cada folio todas las palabras que les vinieron a la mente en un tiempo de dos minutos (Dimitrijévic, 1969)5 cronometrados por el encuestador. Se usó un cronómetro para alcanzar la precisión del tiempo ajustado a dos minutos al igual que muchos investigadores del Proyecto Panhispánico de Disponibilidad léxica (PPHDL). La recogida de los datos se hizo mediante el uso de las listas abiertas porque estas ofrecen, según Samper Padilla y Samper Hernández (2006), la posibilidad de

4. $\quad$ De este modo, los diecisiete centros de interés habitualmente más empleados en los estudios de disponibilidad léxica son los siguientes: 01. Partes del cuerpo, 02. La ropa, 03. Partes de la casa (sin los muebles), 04. Los muebles de la casa, 05. Alimentos y bebidas, 06. Objetos colocados en la mesa para la comida, 07. La cocina y sus utensilios, 08. La escuela: muebles y materiales, 09. Iluminación, calefacción y medios de airear un recinto, 10. La ciudad, 11 El. campo, 12. Medios de transporte, 13. Trabajos del campo y del jardín, 14. Los animales, 15. Juegos y distracciones, 16. Profesiones y oficios y 17. Los colores. Se ha añadido este último ámbito temático con fines investigativos concretos, sobre todo en muestras de Español como Lengua Extranjera. Véase Sandu, 2014; Serfati, 2017,2018 y 2019.

5. La recolección de los datos se hizo de forma escrita, por lo que en tan solo dos minutos muchos alumnos completaron las veintiséis casillas vacías que tienen en cada centro de interés. Incluso, en muchas ocasiones, el informante sigue escribiendo en el reverso de cada folio. 
La evolución del léxico disponible en español como lengua extranjera en aprendices marroquíes hacer análisis sociolingüísticos y determinar el número de vocablos diferentes que puede aportar cada alumno en cada centro de interés.

\subsection{Procedimiento}

Antes de informar y calcular el vocabulario evocado por los encuestados, se aplicaron los diferentes criterios de edición comunes al Proyecto Panhispánico de Disponibilidad Léxica (PPHDL), propuestos por el profesor Samper Padilla (1998) y adoptados en la reunión de San Millán en 2003. ${ }^{6}$ Se aplicaron también los criterios específicos propuestos por Samper Hernández (2002) para estudiar el léxico disponible en español como lengua extranjera.

Por último, después de la limpieza del corpus recogido, en la etapa de la edición y del procesamiento informático de los datos, se obtuvo, entre otros índices, el de disponibilidad, el de frecuencia, el de aparición y el de frecuencia acumulada. Para ello, se utilizó el programa LexiDisp, ${ }^{7}$ creado por Moreno Fernández y García de las Heras, con la asesoría de Moreno Fernández y Benítez Pérez. Este programa, patrocinado por la Asociación de Lingüística y Filología de América Latina (ALFAL), el Instituto Cervantes y la Universidad de Alcalá, aplica la fórmula matemática de los estudiosos mexicanos López Chávez y Strassburger Frías (1987).

\section{Resultados cuantitativos generales}

El análisis de los descriptores estadísticos obtenidos tras el conteo de la disponibilidad léxica de los aprendices marroquíes de español como lengua extranjera en la enseñanza secundaria y universitaria permite conocer el número tanto de palabras como de vocablos diferentes producidos a partir de los centros de interés contemplados por el Proyecto Panhispánico. En la tabla 2 se presentan los resultados globales de disponibilidad léxica en cada uno de los grupos investigados.

\begin{tabular}{|c|c|c|c|c|}
\cline { 2 - 5 } \multicolumn{1}{c|}{} & $\begin{array}{c}\text { Número de } \\
\text { palabras }\end{array}$ & $\begin{array}{c}\text { Vocablos } \\
\text { diferentes }\end{array}$ & $\begin{array}{c}\text { Promedio de palabras } \\
\text { de cada informante }\end{array}$ & $\begin{array}{c}\text { Promedio de vocablos } \\
\text { diferentes por centro } \\
\text { de interés }\end{array}$ \\
\hline Secundaria & 39462 & 4316 & 112,7 & 253,8 \\
\hline Universidad & 49353 & 3742 & 141 & 220,1 \\
\hline Porcentaje de incremento & $25 \%$ & $15,3 \%$ & $25 \%$ & $15,3 \%$ \\
\hline
\end{tabular}

Tabla 2. Datos globales por niveles educativos. Fuente: elaboración propia.

$\overline{6 .} \quad$ Se trata del II Encuentro Internacional de Disponibilidad Léxica que se celebró en San Millán de la Cogolla, en el año 2003

7. Para más información sobre ese programa, se puede consultar el Manual de usuario de Moreno Fernández en http://www. linguas.net/Proyectos/LexiDisp/tabid/73/language/es-ES/Default.aspx. 
La evolución del léxico disponible en español como lengua extranjera en aprendices marroquíes

En la Tabla 2 aparece el número total de respuestas recogidas por los dos niveles educativos, el número de vocablos diferentes y el promedio de respuestas por informante, resultado de dividir el número total de palabras entre el número de informantes inventariados. Igualmente, se refleja el promedio de vocablos diferentes por centros de interés, resultado de dividir el número total de vocablos diferentes entre los 17 ámbitos temáticos que integran el test asociativo que constituye la encuesta, y diligenciado cada uno de ellos, como ya se ha dicho, en un tiempo límite de dos minutos en el que el informante escribe todas aquellas unidades léxicas que surgen relativas a los mismos.

Como puede apreciarse, la producción léxica de los estudiantes universitarios es superior a la del nivel secundario. Además, esta supremacía puede explicarse también por el hecho de que los estudiantes de universidad poseen una mayor madurez léxica ${ }^{8}$ que los alumnos de secundaria. Los resultados registrados revelan que, en el total de palabras actualizadas, los estudiantes de universidad emiten un promedio del $25 \%$ más de respuestas que los alumnos de secundaria e, igualmente, un 15,3\% más de vocablos por campo asociativo. Sumado a esto, el porcentaje de incremento en el promedio de respuestas de cada informante de universidad es del $25 \%$, más que el de secundaria.

\section{Resultados globales en los centros de interés: análisis y discusión de los descriptores estadísticos}

\subsection{Producción de palabras}

El número de palabras es un dato dependiente del tamaño muestral, es decir, es un dato que engloba todas las respuestas actualizadas por los encuestados e incluso aquellas que están repetidas. En la tabla 3 se muestran los resultados de los campos léxicos en cada uno de los grupos cotejados para ofrecer una visión más amplia sobre la productividad de los informantes, así como el crecimiento léxico del nivel universitario respecto al secundario.

\begin{tabular}{|c|c|c|c|c|}
\hline Rango & Centros de interés & $\begin{array}{c}\text { Total de palabras } \\
\text { (secundaria) }\end{array}$ & $\begin{array}{c}\text { Total de palabras } \\
\text { (universidad) }\end{array}$ & $\begin{array}{c}\text { Porcentaje de } \\
\text { incremento }\end{array}$ \\
\hline 01 & Partes del cuerpo & 2711 & 4001 & 47,8 \\
\hline 02 & La ropa & 1756 & 2318 & 32 \\
\hline 03 & Partes de la casa (sin los muebles) & 2319 & 2853 & 23 \\
\hline 04 & Los muebles de la casa & 2547 & 2565 & 0,7 \\
\hline 05 & Alimentos y bebidas & 3191 & 3234 & 1,3 \\
\hline 06 & Objetos colocados en la mesa para & 1366 & 1883 & 37,8 \\
\hline 07 & La cocina y sus utensilios & 2014 & & 4,8 \\
\hline
\end{tabular}

8. Según Cuba Vega (2016), la madurez léxica es «la capacidad que permite al hablante manipular su léxico fundamental — básico + disponible - , para utilizar el mayor número posible de vocablos, y palabras y vocablos de contenido nocional en un texto, lo que favorecerá una mejor expresión del contenido de su mensaje» (pp. 82-83). 
La evolución del léxico disponible en español como lengua extranjera en aprendices marroquíes

\begin{tabular}{|c|c|c|c|c|}
\hline 08 & La escuela: muebles y materiales & 3490 & 3462 & $-0,8$ \\
\hline 09 & $\begin{array}{c}\text { Iluminación, calefacción y medios } \\
\text { de airear un recinto }\end{array}$ & 1640 & 1695 & 3,3 \\
\hline 10 & La ciudad & 3428 & 3909 & 14 \\
\hline 11 & El campo & 2081 & 3045 & 46,3 \\
\hline 12 & Medios de transporte & 2308 & 2626 & 13,7 \\
\hline 13 & Trabajos del campo y del jardín & 1589 & 2116 & 33,1 \\
\hline 14 & Los animales & 1739 & 3635 & 9 \\
\hline 15 & Juegos y distracciones & 2319 & 2392 & 5,1 \\
\hline 16 & Profesiones y oficios & 2882 & 4508 & 44 \\
\hline 17 & Los colores & 2082 & 2999 & $\mathbf{2 5}$ \\
\hline
\end{tabular}

Tabla 3. Total de palabras e incremento léxico por centro de interés. Fuente: elaboración propia.

Como puede observarse en la Tabla 3, la universidad supera a la secundaria en el total de palabras emitidas en casi todos los centros de interés - excepto el 08. La escuela: muebles y materiales_-, aunque en distintas cuantías. Los centros de interés con mayor número de palabras coinciden en ambos grupos educativos, y estos son el 01. Partes del cuerpo, el 10. La ciudad y el 16. Profesiones y oficios; y también coinciden los menos productivos en ambos niveles: 13. Trabajos del campo y del jardín, 09. Iluminación, calefacción y medios de airear un recinto, 06. Objetos clocados en la mesa para la comida y 02 . La ropa.

En cuanto al número de respuestas emitidas, el porcentaje de crecimiento léxico en universidad es evidente en todos los campos nocionales, siendo los más elevados en orden decreciente los centros 16. Profesiones y oficios, 01. Partes del cuerpo, 11. El campo y 17. Los colores. Por el contrario, el decrecimiento de respuestas mencionadas en dicho nivel se produce en los centros 08. La escuela: muebles y materiales, 04. Los muebles de la casa, 05. Alimentos y bebidas y 15. Juegos y distracciones.

\subsection{Producción de vocablos diferentes}

En la tabla 4 se incluye el número de unidades diferentes, lo que realmente supondría un verdadero indicador de la riqueza léxica ${ }^{9}$ entre ambos grupos de contraste.

9. Cuba Vega (2016) la define como «grado de madurez y dominio léxico general de un hablante, medidos en la producción de sus textos, orales o escritos, por índices estadísticos como el porcentaje de vocablos, el intervalo de aparición de palabras de contenido nocional y el porcentaje de vocablos de contenido nocional» (p. 82). 


\begin{tabular}{|c|c|c|c|c|}
\hline Rango & Centro de interés & $\begin{array}{c}\text { Total de } \\
\text { vocablos } \\
\text { diferentes } \\
\text { (secundaria) }\end{array}$ & $\begin{array}{c}\text { Total de vocablos } \\
\text { diferentes (universidad) }\end{array}$ & $\begin{array}{c}\text { Porcentaje de } \\
\text { incremento }\end{array}$ \\
\hline 01 & Partes del cuerpo & 240 & 136 & $-43,3$ \\
\hline 02 & La ropa & 142 & 129 & $-9,1$ \\
\hline 03 & Partes de la casa (sin los muebles) & 180 & 126 & -30 \\
\hline 04 & Los muebles de la casa & 228 & 180 & -21 \\
\hline 05 & Alimentos y bebidas & 249 & 256 & 2,8 \\
\hline 06 & Objetos colocados en la mesa para & 112 & 134 & 19,6 \\
\hline 07 & la comida & & & 2,2 \\
\hline 08 & La cocina y sus utensilios & 225 & 230 & $-29,9$ \\
\hline 09 & Iluminación, calefacción y medios & 194 & 255 & $-31,4$ \\
\hline 10 & de airear un recinto & & 133 & $-18,2$ \\
\hline 11 & La ciudad & 500 & 409 & $-3,4$ \\
\hline 12 & El campo & 437 & 422 & $-56,6$ \\
\hline 13 & Medios de transporte & 219 & 95 & $-16,6$ \\
\hline 14 & Trabajos del campo y del jardín & 318 & 265 & $-11,2$ \\
\hline 15 & Los animales & 214 & 190 & $-3,1$ \\
\hline 16 & Juegos y distracciones & 285 & 276 & 20,4 \\
\hline 17 & Profesiones y oficios & 347 & 418 & 41,9 \\
\hline & Los colores & 62 & 88 & $\mathbf{- 1 3 , 3}$ \\
\hline
\end{tabular}

Tabla 4. Total de vocablos diferentes e incremento léxico por centro de interés. Fuente: elaboración propia.

Estos resultados muestran que no existe una proporción entre los totales de palabras y vocablos diferentes recogidos para cada uno de los centros de interés, por lo que, según Gómez Molina y Gómez Devís (2004), «no se cumple la máxima: a mayor número de respuestas, mayor número de palabras diferentes» (p. 77). No obstante, en cuanto a vocablos o unidades diferentes, la secundaria supera a la universidad en 12 centros de interés: 01. Partes de cuerpo, 02. La ropa, 03. Partes de la casa ( $\sin$ los muebles), 04. Los muebles de la casa, 08. La escuela: muebles y materiales, 09. Iluminación, calefacción y medios de airear un recinto, 10. La ciudad, 11. El campo, 12. Medios de transporte, 13. Trabajos del campo y del jardín, 14. Los animales y 15. Juegos y distracciones. Esta supremacía de secundaria en cuanto a los vocablos diferentes se debe, en gran parte, al aumento de las asociaciones secundarias que se han conservado en los listados finales con el fin de mantener al máximo la información actualizada por los encuestados. 
La evolución del léxico disponible en español como lengua extranjera en aprendices marroquíes

Asimismo, hay una coincidencia entre ambos niveles en los campos asociativos que presentan un mayor número de unidades diferentes, con la misma posición jerárquica de las listas en el número de respuestas emitidas por los encuestados. Dichos centros de interés son el 10. La ciudad, seguido en la secundaria del 11. El campo, el 08. La escuela: muebles y materiales y el 16. Profesiones y oficios; y en la universidad, se suceden en este orden: 11. El campo, seguido del 16. Profesiones y oficios, el 10. La ciudad y el 15. Juegos y distracciones. Por el contrario, los ámbitos temáticos con un menor número de vocablos diferentes solo presentan igualdad en uno de ellos, siendo en secundaria, en orden creciente, el 17. Los colores, el 06. Objetos colocados en la mesa para la comida, el 02. La ropa y el 03. Partes de la casa ( $\sin$ los muebles); y en universidad, 12. Medios de transporte, que curiosamente es superado en productividad por secundaria en 124 vocablos más; el 03. Partes de la casa ( $\sin$ los muebles) y el 02. La ropa. Estos últimos con una posición de más o menos en ambos grupos de contraste.

Por otro lado, resulta llamativo el porcentaje de crecimiento léxico en relación con los vocablos emitidos en los dos niveles, pues si bien es superior en secundaria, llama la atención que la productividad de vocablos sea a favor de universidad en los centros de interés 17. Los colores, con un 41,9\% más de palabras diferentes que secundaria, 16. Profesiones y oficios (20,4\%), 06. Objetos colocados en la mesa para la comida (19,6 \%), 05 . Alimentos y bebidas (2,8 \%) y 07 . La cocina y sus utensilios (2,2\%).

\subsection{Promedio de respuestas por participante}

Este valor es el resultado de la media de productividad léxica, es decir, se obtiene al dividir el número total de palabras obtenidas en cada centro de interés por el número de informantes que constituyen la muestra para conocer aquellos centros de interés en los que los encuestados tienen más riqueza léxica: aquellos en los que disponen de mayor léxico disponible. En la Tabla 5 se muestra el promedio de respuestas por informante en ambos niveles. Además, se observa el incremento de respuestas de universidad y lo que ello supone en porcentaje con relación al total de respuestas en cada centro.

\begin{tabular}{|c|c|c|c|c|c|}
\hline Rango & Centro de interés & $\begin{array}{c}\text { Secundaria } \\
\mathbf{( 3 5 0 )}\end{array}$ & $\begin{array}{c}\text { Universidad } \\
\mathbf{( 3 5 0 )}\end{array}$ & $\begin{array}{c}\text { Incremento en respuestas } \\
\text { en universidad }\end{array}$ & $\begin{array}{c}\text { Porcentaje de } \\
\text { incremento } \\
\text { en respuestas }\end{array}$ \\
\hline 01 & Partes del cuerpo & 7,7 & 11,4 & 3,7 & 48 \\
\hline 02 & La ropa & 5 & 6,6 & 1,6 & 22,7 \\
\hline 03 & $\begin{array}{c}\text { Partes de la casa } \\
\text { (sin los muebles) }\end{array}$ & 6,6 & 8,1 & 0,1 & 1,3 \\
\hline 04 & $\begin{array}{c}\text { Los muebles de la } \\
\text { casa }\end{array}$ & 7,2 & 7,3 & 0,1 & 1,1 \\
\hline 05 & $\begin{array}{c}\text { Alimentos y } \\
\text { bebidas }\end{array}$ & 9,1 & 9,2 & & 32 \\
\hline
\end{tabular}


La evolución del léxico disponible en español como lengua extranjera en aprendices marroquíes

\begin{tabular}{|c|c|c|c|c|c|}
\hline 06 & $\begin{array}{c}\text { Objetos colocados } \\
\text { en la mesa para la } \\
\text { comida }\end{array}$ & 3,9 & 5,3 & 1,4 & 35,9 \\
\hline 07 & $\begin{array}{c}\text { La cocina y sus } \\
\text { utensilios }\end{array}$ & 5,7 & 6 & 0,3 & 5,2 \\
\hline 08 & $\begin{array}{l}\text { La escuela: } \\
\text { muebles y } \\
\text { materiales }\end{array}$ & 9,9 & 9,8 & $-0,1$ & -1 \\
\hline 09 & $\begin{array}{c}\text { Iluminación, } \\
\text { calefacción y } \\
\text { medios de airear un } \\
\text { recinto }\end{array}$ & 4,6 & 4,8 & 0,2 & 4,3 \\
\hline 10 & La ciudad & 9,7 & 11,1 & 1,4 & 14,4 \\
\hline 11 & El campo & 5,9 & 8,7 & 2,8 & 47,4 \\
\hline 12 & $\begin{array}{l}\text { Medios de } \\
\text { transporte }\end{array}$ & 6,5 & 7,5 & 1 & 15,3 \\
\hline 13 & $\begin{array}{c}\text { Trabajos del campo } \\
\text { y del jardín }\end{array}$ & 4,5 & 6 & 1,5 & 29,6 \\
\hline 14 & Los animales & 4,9 & 10,3 & 5,4 & 10,2 \\
\hline 15 & $\begin{array}{c}\text { Juegos y } \\
\text { distracciones }\end{array}$ & 6,6 & 6,8 & 0,2 & 3 \\
\hline 16 & $\begin{array}{c}\text { Profesiones y } \\
\text { oficios } \\
\end{array}$ & 8,2 & 12,8 & 4,6 & 56 \\
\hline 17 & Los colores & 5,9 & 8,5 & 2,6 & 44 \\
\hline & Total & 111,9 & 140,2 & 28,3 & 25,2 \\
\hline
\end{tabular}

Tabla 5. Promedio de respuestas por informante de los centros de interés. Fuente: elaboración propia.

Los resultados indican que el centro de interés 08. La escuela: muebles y materiales es el más productivo en alumnos de secundaria, ya que cuenta con 9,9 palabras por sujeto, seguido por el 10. La ciudad, con 9,7 palabras y por el 05. Alimentos y bebidas, con 9,1 palabras por encuestado. En cambio, los centros de interés que cuentan con menos respuestas por informante son el 06. Objetos colocados en la mesa para la comida, con 3,9 palabras; el 13. Trabajos del campo y del jardín, con 4,5 palabras; y el 09. Iluminación, calefacción y medios de airear un recinto, con 4,6 palabras por informante.

Por otra parte, el valor más alto en los universitarios aparece en el centro 16. Profesiones y oficios, pues cuenta con 12,8 palabras por informante. Han obtenido valores muy próximos a este los ámbitos temáticos 01 . Partes del cuerpo, con 11,3 y 10. La ciudad con 11,1 palabras por encuestado. Por el contrario, los centros de interés con 
La evolución del léxico disponible en español como lengua extranjera en aprendices marroquíes

menos palabras por participante son 09. Iluminación, calefacción y medios de airear un recinto, con 4,8 palabras, seguido por 06. Objetos colocados en la mesa para la comida, con 5,3 palabras por informante y, por último, 07 . La cocina y sus utensilios, con 6 respuestas.

En lo correspondiente al incremento de respuestas en universidad respecto a secundaria, el primero supera en promedio de respuestas al segundo en casi todos los centros de interés. De esta forma, los campos asociativos con un número superior de respuestas en universidad frente a secundaria son el 14. Los animales, con 5,4 palabras más, y el 16. Profesiones y oficios, con 4,6 respuestas más, además de un mayor porcentaje de incremento léxico, con el $56 \%$. Por el contrario, los campos nocionales que presentan un menor incremento léxico en número de respuestas de estudiantes universitarios cotejados con los alumnos de secundaria son el 08. La escuela: muebles y materiales, con 0,1 respuestas menos, y el porcentaje de incremento léxico más bajo, con el $-1 \%$; seguido del centro 05. Alimentos y bebidas, con 0,1 respuestas más - y también uno de los porcentajes de incremento léxico más bajo, con el 1,1\%—. Entre aquellos con porcentaje de incremento más bajo, además de los citados anteriormente, se destacan los centros 04. Los muebles de la casa, con el 1,3\% de incremento, el 15. Juegos y distracciones, con el $3 \%$, el 09 . Iluminación, calefacción y medios de airear un recinto, con el 4,3\% y 07 . La cocina y sus utensilios, con el 5,2\% de incremento.

\subsection{Grado de densidad léxica}

Este valor es el resultado de dividir el total de palabras en cada uno de los campos léxicos por el número de vocablos diferentes correspondiente. Este índice describe la naturaleza cuantitativa de cada ámbito temático independientemente y propicia el establecimiento de comparaciones entre los centros del corpus (López Morales, 1995). En este sentido, se advierte una relación directa entre la densidad léxica y el número de participantes: a mayor cantidad de participantes mayor cantidad de palabras y, por lo tanto, mayor índice de densidad léxica. Hernández Muñoz (2006) lo definió de esta manera:

El índice de cohesión y la densidad no están libres de la influencia de la variación en el número de informantes que participan en una determinada muestra, ya que el número de palabras diferentes, divisor en ambos casos, tiende a aumentar cuando asciende el número de sujetos, pero no de forma constante, sino solo hasta un determinado punto en el cual el crecimiento de la curva se estabiliza (p. 311).

De este modo, las apreciaciones realizadas sobre los centros de interés, más o menos cohesionados, serán igualmente aplicables desde el punto de vista de la densidad léxica. En este caso, el índice de densidad medio es de 10,8 en los estudiantes de secundaria y llega a un 15,9 en los de universidad. 


\begin{tabular}{|c|c|c|c|c|c|}
\hline Rango & Centro de interés & $\begin{array}{c}\text { Secundaria } \\
(\mathbf{3 5 0}) \\
\end{array}$ & $\begin{array}{c}\text { Universidad } \\
(\mathbf{3 5 0}) \\
\end{array}$ & $\begin{array}{c}\text { Incremento en } \\
\text { universidad }\end{array}$ & $\begin{array}{c}\text { Porcentaje de } \\
\text { incremento } \\
\end{array}$ \\
\hline 01 & Partes del cuerpo & 11,3 & 29,4 & 18,1 & 60,1 \\
\hline 02 & La ropa & 12,3 & 17,9 & 5,6 & 45,5 \\
\hline 03 & $\begin{array}{c}\text { Partes de la casa ( } \sin \text { los } \\
\text { muebles) }\end{array}$ & 12,8 & 22,6 & 9,8 & 76,5 \\
\hline 04 & Los muebles de la casa & 11,1 & 14,2 & 3,1 & 27,9 \\
\hline 05 & Alimentos y bebidas & 12,8 & 12,6 & $-0,2$ & $-1,5$ \\
\hline 06 & $\begin{array}{c}\text { Objetos colocados en la } \\
\text { mesa para la comida }\end{array}$ & 12,2 & 14 & 1,8 & 14,7 \\
\hline 07 & La cocina y sus utensilios & 8,9 & 9,1 & 0,2 & 2,2 \\
\hline 08 & $\begin{array}{c}\text { La escuela: muebles y } \\
\text { materiales }\end{array}$ & 9,5 & 13,5 & 4 & 42,1 \\
\hline 09 & $\begin{array}{l}\text { Iluminación, calefacción y } \\
\text { medios de airear un recinto }\end{array}$ & 8,4 & 12,7 & 4,3 & 51,1 \\
\hline 10 & La ciudad & 6,8 & 9,5 & 2,7 & 39,7 \\
\hline 11 & El campo & 4,7 & 7,2 & 2,5 & 53,1 \\
\hline 12 & Medios de transporte & 10,5 & 27,6 & 17,1 & 62,8 \\
\hline 13 & $\begin{array}{c}\text { Trabajos del campo y del } \\
\text { jardín }\end{array}$ & 4,9 & 7,9 & 3 & 61,2 \\
\hline 14 & Los animales & 8,1 & 19,1 & 11 & 35,8 \\
\hline 15 & Juegos y distracciones & 8,1 & 8,6 & 0,5 & 6,1 \\
\hline 16 & Profesiones y oficios & 8,3 & 10,7 & 2,4 & 28,9 \\
\hline \multirow[t]{2}{*}{17} & Los colores & 33,5 & 34 & 0,5 & 1,4 \\
\hline & Total & 184,2 & 270,6 & 86,4 & 46,9 \\
\hline
\end{tabular}

Tabla 6. Densidad léxica por centro de interés. Fuente: elaboración propia.

Como se puede ver en la Tabla 6, cuantos menos vocablos diferentes haya en un centro de interés, mayor será su densidad léxica y, en consecuencia, mayor grado de repetición de los mismos vocablos diferentes se hallarán. Se puede observar que, en los alumnos de secundaria, los índices de densidad léxica oscilan entre 33,5 y 4,7, respectivamente, y el promedio de todos los centros de interés es de 10,8. En los universitarios los índices tienen exactamente la misma oscilación, pero el promedio de 15,9 es un poco más alto, lo cual indica que los estudiantes universitarios han arrojado más unidades léxicas que los alumnos de secundaria.

En los dos grupos de contraste, el campo semántico con mayor densidad léxica es el 17. Los colores, con el mismo grado de densidad de 33,5 en el grupo de secundaria y de 34 en el de universidad. Se trata, pues, de un área temáticamente uniforme contrastada con todos los campos léxicos estudiados, mientras que los centros 13. Trabajos 
La evolución del léxico disponible en español como lengua extranjera en aprendices marroquíes

del campo y de jardín y 11. El campo, los centros más dispersos en la mayoría de los estudios de disponibilidad léxica, pueden arrojar asociaciones muy diferentes. Estos resultados coinciden con las investigaciones llevadas a cabo con hispanohablantes (Gómez Molina \& Gómez Devís, 2004; Samper Padilla, Bellón Fernández \& Samper Hernández, 2003).

En lo referente al incremento de densidad léxica en estudiantes universitarios respecto a alumnos de secundaria, los primeros superan a los segundos en casi todos los centros de interés. De esta manera, los ámbitos temáticos con mayor grado de densidad léxica en los universitarios frente a los alumnos de secundaria son el 01. Partes del cuerpo, con 18,1 y el 12. Medios de transporte, con 17,1; que también poseen el mayor porcentaje de incremento léxico, con el $62,8 \%$.

Por el contrario, los centros de interés que presentan un menor incremento de densidad léxica en los estudiantes universitarios comparados con los alumnos de secundaria son el 05 . Alimentos y bebidas, con - 0,2 y un porcentaje de incremento de densidad léxica más bajo: el -1,5\%; seguido del centro de interés 17 . Los colores, con 0,5, que también tiene uno de los porcentajes de incremento léxico más bajos, con el 1,4\%. Aparte de estos, destacan otros campos léxicos cuya densidad léxica sigue siendo más baja, tal como es el caso del centro 07 . La cocina y sus utensilios, con un 2,2\% de incremento, el 15. Juegos y distracciones, con un 6,1 \% de incremento y el 06 . Objetos colocados en la mesa para la comida, con un $14,7 \%$ de incremento.

\section{Conclusiones}

El análisis cuantitativo de los datos ha permitido detectar los centros de interés en que los informantes incorporan una mayor disponibilidad y en los que presentan una carencia léxica. Los alumnos de secundaria emitieron un total de 39462 palabras, mientras que el grupo de universitarios escribió 49353 palabras —un $25 \%$ de incremento léxico a favor de los universitarios-. El promedio de palabras indica que la cantidad de respuestas por cada alumno de secundaria es de 112,7 respuestas por informante y 2321,3 unidades léxicas por área asociativa; en los universitarios, por su parte, el promedio es de 141 respuestas por informante y 2903,1 por centro de interés — un 24,2 \% de incremento léxico también a favor de los estudiantes de universidad-.

Asimismo, el incremento léxico observado en los estudiantes universitarios puede deberse a factores de índole psicológica. ${ }^{10}$ Como indicó Hernández Muñoz (2006):

La eficacia a la hora de responder a las encuestas de léxico disponible no debemos atribuirlas exclusivamente a que los alumnos conozcan mayor o menor número de palabras, sino también a la familiaridad que el colectivo de estudiantes tenga con pruebas de este tipo, así como a las estrategias lingüístico-cognitivas que sean capaces de desarrollar en ese momento dado. (p. 330).

Precisamente relacionado con ello, es evidente que los universitarios se han enfrentado con más pruebas escritas de esta categoría que los alumnos de secundaria y, por lo tanto, su experiencia les permite desarrollar nuevas estrategias de actualización léxica (Valenzuela Castellanos, Pérez Villalobos, Bustos \& Salcedo Lagos,

$\overline{10 .} \quad$ También está la variable evolutiva, contextual o cultural. 
2018).

De todo lo dicho, y manera de comentario final, se desprende que los estudios de disponibilidad léxica tienen como objetivo evaluar la competencia léxica de los hablantes de una lengua (Marconi, 2000), es decir, evaluar las estrategias que se refieren tanto a las relaciones entre palabras como a las expresiones dentro del sistema lingüístico. Además, la disponibilidad léxica, en tanto disciplina científica, se considera una herramienta de índole multidisciplinar, es decir que no es exclusivamente lingüística, sino que unifica el estudio del léxico desde diferentes y múltiples perspectivas. 


\section{Referencias bibliográficas}

1. Aabidi, L. (2020). La disponibilidad léxica en español de alumnos marroquíes de enseñanza media: resultados generales. Philologica Canariensia, 26, 1-19.

2. Blanco-San Martín, L. P. \& Ferreira Cabrera, A. A. (2018). Léxico disponible en tres centros de interés de aprendices de español como lengua extranjera. Íkala: Revista de Lenguaje y Cultura, 23(3), 505-517.

3. Camarena Ortiz, E. D. (2010). La interlengua en el léxico disponible de un grupo de alumnos de portugués en México. En I. Moskowich-Spiegel Fandiño, B. Crespo-García, I. Lareo \& P. Lojo (Eds.), Language Windowing through Corpora. Visualización del lenguaje a través de corpus (pp. 145-155). La Coruña: Universidad de la Coruña.

4. Carcedo González, A. (2000). Disponibilidad léxica en español como lengua extranjera: el caso finlandés (estudio del nivel preuniversitario y cotejo con tres fases de adquisición). Turku: Universidad de Turku.

5. Cuba Vega, L. E. (2016). Sexo y vocabulario: relación en escolares habaneros. Enunciación, 2(1), 80-90.

6. Dimitrijévic, N. R. (1969). Lexical Availability. A New Aspect of the Lexical Availability of Secondary School Children. Heidelberg: Julius Gross Verlag.

7. Fernández Leyva, H. (2013). La selección y ocurrencias del vocabulario por centros de interés en el manual Español Para Todos 1. (Memoria de máster). Santiago de Cuba: Universidad de Oriente.

8. Fernández-Merino Gutiérrez, P. V. (2013). Disponibilidad léxica de inmigrantes en Castilla y León (Tesis doctoral). Valladolid: Universidad de Valladolid.

9. Gallego Gallego, D. J. (2014). Léxico disponible de estudiantes de Español como Lengua Extranjera en la Comunidad de Madrid. (Tesis doctoral). Alcalá de Henares: Universidad de Alcalá.

10. Gómez Molina, J. R. \& Gómez Devís, M. B. (2004). La disponibilidad léxica de los estudiantes preuniversitarios valencianos. Estudio de estratificación sociolingüística. València: Universitat de València.

11. González Fernández, J. (2013). La disponibilidad léxica de los estudiantes turcos de Español como Lengua Extranjera. MarcoELE: Revista de Didáctica ELE, 16, 1-14.

12. Gougenheim, G., Michéa, R., Rivenc, P. \& Sauvageot, A. (1956). L'élaboration du français élémentaire. Étude sur l'établissement d'un vocabulaire et d'une grammaire de base. Paris: Didier.

13. Hernández Muñoz, N. (2006). Hacia una teoría cognitiva integrada de la disponibilidad léxica. El léxico disponible de los estudiantes castellano-manchegos. Salamanca: Ediciones Universidad de Salamanca.

14. Hidalgo Gallardo, M. (2018). La disponibilidad léxica como método de detección del vocabulario y de su selección en los manuales: aplicación en una muestra de estudiantes sinohablantes de ELE (Tesis doctoral). Jaén: Universidad de Jaén.

15. Jiménez Berrio, F. (2013). Léxico disponible de inmigrantes escolares no hispanohablantes. Pamplona: Servicio de Publicaciones de la Universidad de Navarra.

16. Jing, L. (2006). Estudio de disponibilidad léxica de los estudiantes chinos de español como lengua extranjera. 
La evolución del léxico disponible en español como lengua extranjera en aprendices marroquíes

(Memoria de máster). Alcalá de Henares (Madrid): Universidad de Alcalá de Henares.

17. López Chávez, J. \& Strassburguer Frías, C. (1987). Otro cálculo del índice de disponibilidad léxica. En Presente y Perspectiva de la investigación computacional en México. Actas del IV Simposio de la Asociación de Lingüística Aplicada. México: Universidad Nacional Autónoma de México.

18. López González, A. M. (2010). La evaluación del desarrollo de la competencia léxica en L2 por medio de la disponibilidad léxica. Revista Electrónica de Didáctica ELE (redELE), 18, 1-13.

19. López González, A. M. (2013). Desarrollo de los estudios de disponibilidad léxica en Español Lengua Extranjera (ELE). En I. Contreras \& M. Narciso (Ed. lit.), La enseñanza del Español como LE/L2 en el siglo XXI (pp. 397-408). Jaén: ASELE.

20. López González, A. M. (2014). Disponibilidad léxica. Teoría, método y análisis. Varsovia: Universidad de Łódz.

21. López Morales, H. (1973). Disponibilidad léxica en escolares de San Juan. Puerto Rico: MS.

22. López Morales, H. (1978). Frecuencia léxica, disponibilidad y programación curricular. En H. López Morales (Ed.), Aportes de la Lingüística a la Enseñanza del Español como Lengua Materna, número especial del Boletín de la Academia Puertorriqueña de la Lengua Española (BAPLE), 6(1), 73-86.

23. López Morales, H. (1979). Disponibilidad léxica y estratificación socioeconómica. En H. López Morales (Ed.), Dialectología y Sociolingüística. Temas Puertorriqueños (pp. 173-181). Madrid: Hispanova de Ediciones. 24. López Morales, H. (1995). Los estudios de disponibilidad léxica: pasado y presente. Boletín de filología de la Universidad de Chile, 35, 245-259.

25. López Rivero, E. (2008). Estudio de disponibilidad léxica en 43 estudiantes de ELE (Memoria de máster). Madrid: Universidad Antonio de Nebrija.

26. Marconi, D. (2000). La competencia léxica. Madrid: Visor.

27. Medina Arejita, E. T. (2009). Las nociones especificas del PCIC y la disponibilidad léxica como instrumento de selección del vocabulario. El caso de 43 estudiantes alemanes de español en Berlín (Memoria de máster). Universidad Internacional Menéndez Pelayo: Instituto Cervantes.

26. Mendoza Puertas, D. (2018). El léxico disponible de 82 estudiantes coreanos de Español como Lengua Extranjera. MarcoELE. Revista de Didáctica ELE, 26, 1-25.

27. Michéa, R. (1953). Mots fréquents et mots disponibles. Un aspect nouveau de la statistique du langage. Les Langues Modernes, 47, 338-344.

28. Pedroni Torres, T. (2015). El léxico disponible de los profesores de E/LE en formación en la ciudad de São Paulo (Brasil) (Tesis doctoral). Salamanca: Universidad de Salamanca.

30. Pérez Serrano, M. (2009). Estudio de disponibilidad léxica en estudiantes de E/LE en los centros de interés «Medios de transporte»y «Profesiones y oficios» (Memoria de máster). Instituto Cervantes: Universidad Internacional Menéndez Pelayo.

31. Rubio Lastra, M. (2020). Evaluación de la suficiencia léxica de estudiantes taiwaneses universitarios de ELE 
La evolución del léxico disponible en español como lengua extranjera en aprendices marroquíes

A1 por medio de la disponibilidad léxica y el Plan Curricular del Instituto Cervantes. Linred: Lingüística en la Red, 17, 1-42.

32. Samper Hernández, M. (2002). Disponibilidad léxica en alumnos de español como lengua extranjera. Málaga: ASELE.

33. Samper Padilla, J. A. (1998). Criterios de edición del léxico disponible: sugerencias. Lingüística, 10, $311-333$. 34. Samper Padilla, J. A., Bellón Fernández, J. J. \& Samper Hernández, M. (2003). El proyecto de estudio de la disponibilidad léxica en español. En G. Wotjak (Coord.), Pautas y pistas en el análisis del léxico hispano (americano) (pp. 27-140). Fráncfort-Madrid: Vervuert-Iberoamericana.

35. Samper Padilla, J. A. \& Samper Hernández, M. (2006). Aportaciones recientes de los estudios de disponibilidad léxica. Lynx: Panorámica de Estudios Lingüísticos, 5, 5-95.

36. Sandu, B. (2014). Léxico disponible de alumnos rumanos que aprenden español como lengua extranjera en centros escolares bilingües rumano-españoles (Tesis doctoral). Las Palmas de Gran Canaria: Universidad de Las Palmas de Gran Canaria.

37. Sánchez-Saus Laserna, M. (2016). Léxico disponible de los estudiantes de español como lengua extranjera en las universidades andaluzas. Sevilla: Editorial Universidad de Sevilla.

38. Serfati, M., \& Aabidi, L. (2013). Disponibilidad léxica de ELE en Marruecos (Niveles de Secundaria y Enseñanza Superior en la región Souss Massa Drâa). Agadir (Marruecos): Universidad de Ibn Zohr.

39. Serfati, M. (2016). La disponibilidad léxica de estudiantes marroquíes de nivel universitario: resultados generales. Philologica Canariensia, 22, 105-116.

40. Serfati, M. (2017). Incidencia cuantitativa del factor lengua materna en la disponibilidad léxica de estudiantes marroquíes de Español como Lengua Extranjera (nivel universitario). Estudios Interlingüísticos, 5, 121-145.

41. Serfati, M. (2018). El léxico disponible en estudiantes marroquíes de ELE (nivel universitario): incidencia de la variable «sexo» y su correlación con el «curso académico». Beoiberística, 2(1), 153-180.

42. Serfati, M. (2019). Disponibilidad léxica y la selección léxica en los manuales de ELE en Marruecos (nivel de enseñanza secundaria en la región Souss-Massa). Revista de la Asociación Europea de Profesores de Español: El español por el mundo, 2, 311-324.

40. Sifrar kalan, M. (2009). Disponibilidad léxica en español como lengua extranjera: el cotejo de las investigaciones en Eslovenia, Salamanca y Finlandia. Verba Hispánica: anuario del Departamento de la Lengua y Literatura Españolas de la Facultad de Filosofía y Letras de la Universidad de Ljubljana, 17, 165-182.

41. Valenzuela Castellanos, M. F., Pérez Villalobos, M. V., Bustos, C. \& Salcedo Lagos, P. (2018). Cambios en el concepto aprendizaje de estudiantes de pedagogía: análisis de disponibilidad léxica y grafos. Estudios Filológicos, $61,143-173$. 